\title{
STUDY OF AEROSOL CHARGING IN ELECTRO-AEROSOL GENERATOR
}

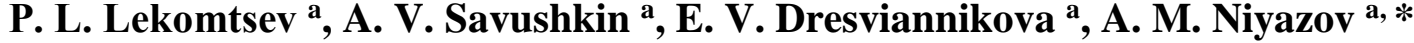 \\ ${ }^{a}$ Federal State Educational Institution Izhevsk State Agricultural Academy, 11 Studencheskaya st, Izhevsk, 426069, Russia; \\ e-mails: lekomcev@ya.ru,dresva@yandex.ru,*niazam@ya.ru
}

Received: 02.08.2017 / Accepted: 15.09.2017 / Revised: 07.11.2017 / Available online: 15.12.2017

DOI: $10.1515 /$ jaes-2017-0016

KEY WORDS: electro-aerosol, electric field intensity, convection current, electric charging, bulk charge

\begin{abstract}
:
The urgency of the study is conditioned by the necessity of perfection of the methods of electro-aerosol disinfection and disinsection of the air and the surfaces of premises, medical and preventive treatment of animals, and plant protection. The objective of the paper is aimed at revealing the regularities of electro-physical processes when charging an aerosol in electro-aerosol generators, improvement of the methods of electro-aerosol generation. The leading approach to the research of this problem is mathematical modelling of electrotechnical, dynamic and kinetic processes in electro-aerosol generators, allowing revealing the basic regularities of electroaerosol generation. Experimental studies are aimed at assessment of the main characteristic of the electro-aerosol generator convection current. As a result of the studies, a mathematical model of electro-aerosol generation with the mechanical method of atomization was obtained. The regularities of charging a fluid thread, arising at the edge of the generator, when applying electric field. The configuration of the electric field of cylindrical electrodes, taking into account the influence of the bulk charge of the electroaerosol cloud, was considered. Electrostatic pressure of the electric field of the generator on fluid streams was taken into consideration. An equation for determining the average radius of drops of the electro-aerosol during mechanical atomization by the perforated cage was obtained. The mathematical model of electro-aerosol generation is obtained in the form of the equation of convection current, being one of the basic characteristics of the process of electro-aerosol generation, allowing defining the efficiency of electrization of aerosol particles and the degree of their fragmentation. Theoretical calculations are verified experimentally. Materials of the paper can be useful for the researchers, post-graduate students, engineers, engaged in designing, manufacturing and exploiting electro-aerosol equipment.
\end{abstract}

\footnotetext{
* Corresponding author: A. M. Niyazov, Associate Professor, Candidate of Engineering Sciences, e-mail: niazam@ ya.ru
} 


\section{INTRODUCTION}

Specialization and concentration of the livestock sector have led to establishing large-scale complexes of industrial type with a high concentration of livestock number on the limited territories. Manufacturing technologies of livestock maintenance significantly increase the efficiency of manufacture of livestock products, but, at the same time, create favourable environment for the spread of pathogenic microorganisms.

The sources of pathogenic microorganisms in livestock premises are sick animals, microbe-carriers animals, as well as remnants of animals' life activity - dung, urine, gases, products of dermal and lung respiration (Blyumin and Astapov, 1987; Borodin et al, 2004; Yarnyh, 1972). Getting to aerial environment, microorganisms form a large amount of a bacterial aerosol, which settles on the surfaces of livestock premises, is disseminated all over the territory of the complex and beyond it.

Vast dissemination of the air creates the danger of aerogenic spreading of the pathogenic microflora from one production building to another. Under these circumstances, a massive coverage of livestock with infectious diseases is possible, which results in huge losses in products.

In this connection, the role of sanitary-veterinary measures significantly increases: disinfection of the air and surfaces of premises, medical and preventive treatment of animals. Chemical substances of disinfection have become the most widespread in veterinary practice.

The effectiveness of the influence of chemical substances depends on the concentration of their solutions, temperature and exposition. For practical application, the chemical substances that possess a reliable bactericidal effect, that are easily soluble in water, do not have unpleasant smell, do not cause corrosion to metal structures, and are nontoxic for the human being and animals are the most acceptable.

Treatment of livestock premises using traditional methods - the wet method and spraying - is very labor consuming and is connected with bulky disinfection equipment and a high consumption of disinfectants. Besides, during treatment, chemical substances are brought out beyond livestock premises and, thus, they contaminate environment.

A number of scientific studies and results of veterinary practice showed that the method of application of chemical and biological substances in an aerosol form increases significantly the efficiency of veterinary measures. Aerosol disinfection possesses a number of advantages compared to the wet one, consisting, according to (Blyumin and Astapov, 1987; Yarnyh, 1972; 1985), in a $3 \ldots 5$ - time reduction of disinfection substances consumption, labor conditions improvement, in the possibility of mechanization and automatization of the disinfection process, in a reduction of a corrosive effect on technological equipment. During aerosol treatment, simultaneously with surface disinfection, decontamination of the air of livestock premises takes place Blyumin, Astapov, 1987; Zakomyrdin, Visnapuu, 1977).

Attachment of electric charge to the aerosol particles allows qualitatively improving the technology of agricultural facilities treatment.

In the electrically charged aerosol, compared to the uncharged one, additional forces of particle interaction occur - electrostatic dispersion and mirror imaging. Electrical forces act in all directions, which enables equalization of concentration by the volume of premises with a simultaneous increase of the aerosol precipitation rate. The direction and the intensity of the forces depend on the size, value and polarity of the charge, particle concentration and treatment volume, which opens up new opportunities for managing the processes of dissemination and precipitation of the electro-aerosol (Borok, 1974; Borodin and Lekomtsev, 2006; Dondokov and Tumureev, 1976; Dunsky and Kitaev, 1960; Law, 1975).

During inhalation chemotherapy, the aerosol provides a more precise dosage of medication for animals; thus, different parts of the animal's respiratory tract can be treated. The influence of the negatively-charged aerosols on the organism is longer than that of ordinary aerosols, and a higher concentration of them is accumulated in the blood and tissues.

However, insufficient development of the scientific base of the engineering calculation and designing of electro-aerosol generators hinders wide application of this method in agriculture.

The process of aerosol obtainment consists in dispersion of a fluid into tiny drops and attachment of electric charge to the drops. Hydraulic, pneumatic, mechanical, ultrasonic, electric, acoustic, etc. methods of fluid dispersion are more widely spread (Borodin et al, 1967; Green and Lane, 1972; Pazhi and Galustov, 1984). The mechanical method of dispersion possesses a number of advantages.

Mechanical atomization is carried out by rotating mechanisms of disc, cone, bowl, etc. type. Fluid in the field of centrifugal forces spreads over the surface of an atomizer, acquires kinetic energy and falls down from the edge in the form of separate drops, thin threads or film. The nature of atomization is in strong dependence on the fluid consumption and the linear velocity of disc rotation. Disc generators, compared to other types - hydraulic and pneumatic ones - have the atomization, approximated to the monodispersed one (the variation coefficient is less than 0.3), allow changing the drop sizes in a wide range, and are not cluttered with insoluble impurities of the atomized fluids (Borodin and Lekomtsev, 2005; Dunsky and Nikitin, 1982; 
Savushkin and Lekomtsev, 1991; Bals, 1970; Frost, 1974; Hinze and Milborn, 1950).

At present, there are several well-known methods of particle electrization (Vereshchagin et al, 1974; Dunsky, 1966; Makalsky, 1991): charging by means of mechanical, chemical or thermal electrization; ionic electrization - owing to precipitation of ions on the particle surface; charging based on the phenomenon of electrostatic induction, that is, charge separation in the electrostatic field. Ionic electrization and charging in the electrostatic field are mostly widely spread.

Particle electrization by means of precipitation of ions on their surfaces is usually realized in the field of corona discharge. This is sufficiently effective method of charging, allowing obtainment of high particle charges independently of their physical properties. Ion precipitation of the particle surface occurs by virtue of ion diffusion and during collision of a particle and ions, moving along the field lines to its surface. To obtain highest charges, the particle must be in the field of corona discharge for a significant amount of time. When charging aerosols, this peculiarity leads to construction complexities since with the increase of the charging period, particle precipitation in the charger takes place.

Analysis of a number of studies (Borok, 1974; Vereshchagin et al, 1974; Dunsky, 1966; Lekomtsev, 2006; Makalsky, 1991) demonstrates that charging aerosol particles in the electrostatic field has a number of advantages over their charging in the field of corona discharge: it is technically easier to realize owing to a combination of the dispersion process and fluid charging in space and in time; it requires lower voltage and charging current, consequently, it is more energy-conserving; it does not pollute environment with nitrogen oxides and ozone, forming in corona discharge.

\subsection{Research objective}

The research objective consists in development of the mathematical model of electro-aerosol generation by the mechanical method, allowing revealing the regularities of electro-physical processes during aerosol charging in electroaerosol generators.

\subsection{Research tasks}

It is necessary to obtain a mathematical expression for calculation of convection current of the electro-aerosol generator, taking into account the charging of the elongated fluid thread in the electrostatic field. It is expedient to experimentally verify the resulted model.

\section{MATERIALS AND METHODS}

Mathematical methods of calculation of electrotechnical, dynamic and kinetic processes in electro-aerosol generators were used.
Experimental studies are aimed at the assessment of the basic characteristic of the electro-aerosol generator - convectional current. Generator PMEG (pneumomechanical, electro-aerosol generator (Savushkin and Lekomtsev, 1991; Borodin and Lekomtsev, 2005)) was used as a model one. The PMEG generator has a radius of an atomizing element equal to $0.16 \mathrm{~m}$. At a rotation frequency of $\omega=837.7 \mathrm{~s}^{-1}$ and a volume fluid flow rate of $Q_{f}=1 \ldots 9 \mathrm{ml} / \mathrm{s}$, the generator provides a median radius of the aerosol of $d=20 \ldots 40 \mu \mathrm{m}$.

For measuring the generator convection current, a method of a "large collector" (Savushkin and Lekomtsev, 1991; Savushkin et al, 1991) was used, the essence of which consists in the following. Electro-aerosol generator 1 was placed inside large collector 2 (figure 1), grounded through measuring device 3 . The collector is surrounded by grounded screen-electrode 4 . Aerosol particles precipitate on the inner surface of the collector, and the current, numerically equal to the summary charge of all drops, produced by the generator per time unit, flows in the device circuit.

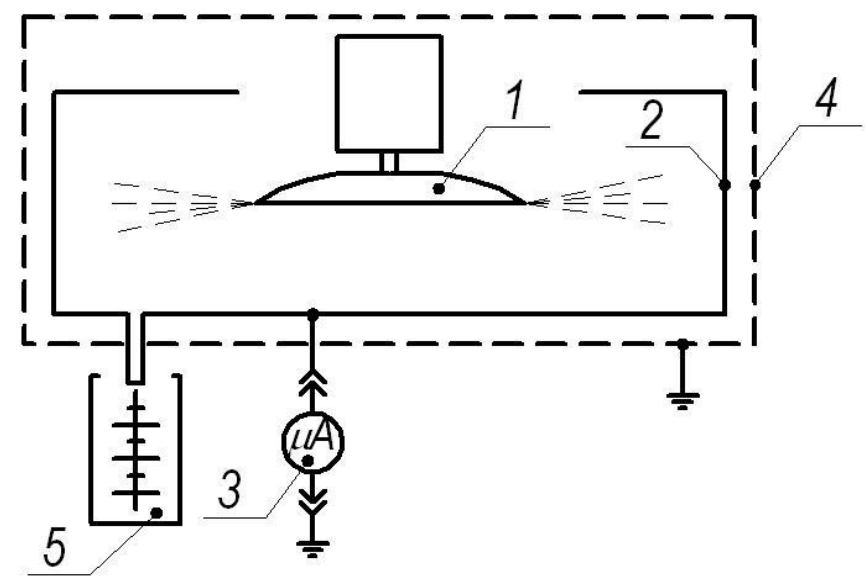

Figure 1. Scheme of measuring average-mass charge of electroaerosol particles

1 - electro-aerosol generator; 2 - collector; 3 - microammeter; 4 - grounded screen-electrode; 5 - measuring container.

Convection current is measured by the microammeter or galvanometer of the magnetoelectric system. The voltage of generator charging is measured by the kilovoltmeter.

The fluid consumption is set by the metering device or determined as a ratio of $m / t$, where $m$ is fluid mass, accumulated in measuring container 5 in time $t$.

\section{RESULTS AND DISCUSSION}

The process of formation of the electrically charged aerosol consists in forming a fluid film by special devices, with its subsequent charging and disintegration in the electric field into separate charged drops. 
When atomizing fluids by mechanical generators, the fluid torus, from which separate threads are drawn out, forms at the edge of the disc (Dunsky et al, 1973; Hinze and Milborn, 1950). At some distance from the disc edge, these threads disintegrate into similar in sizes drops.

Electrically charged drops, moving in the electric field and the air stream of the generator, create the charge transfer current convection current. Convection current is one of the basic characteristics of the electro-aerosol generation process, which determines the efficiency of electrization of aerosol particles and the degree of their fragmentation.

In the moving medium, the total current through some surface can be written as:

$I=\frac{d q}{d t}=\frac{d \sigma_{s} S}{d t}=\sigma_{s} \frac{d S}{d t}+S \frac{d \sigma_{s}}{d t}$,

where: $\sigma_{s}$ - charge density of surface $S, \mathrm{C} / \mathrm{m}^{2}$.

In expression (1), product $\sigma_{s} \frac{d S}{d t}$ characterizes macromovement of surface charges, that is, convection current; at that, $d S$ represents the rate of new surface formation, for instance, in the form of the film, threads or separate drops. Value $\frac{d \sigma_{s}}{d t}$ - the rate of charge density change on surface $S$ - determines conduction current or bias current in the medium. Thus, convection current is determined only by the first term, standing in the right part of equation (1).

Thus, it is possible to write down the following:

$$
I_{k}=\sigma_{s} \frac{d S}{d t},
$$

Let us consider the electro-aerosol generation model under the following assumptions: the fluid thread is supposed to be a cylinder with length $l$, the diameter of which reduces from the edge of the hole to its periphery (figure 2); the cylinder is charged in the uniform field with intensity $E$; the cylinder axis can be directed along the field or perpendicular to $E$; contact resistance of the thread can be neglected, the surface conductance is supposed to be zero; the cylinder is approximated by the elongated hemisphere.

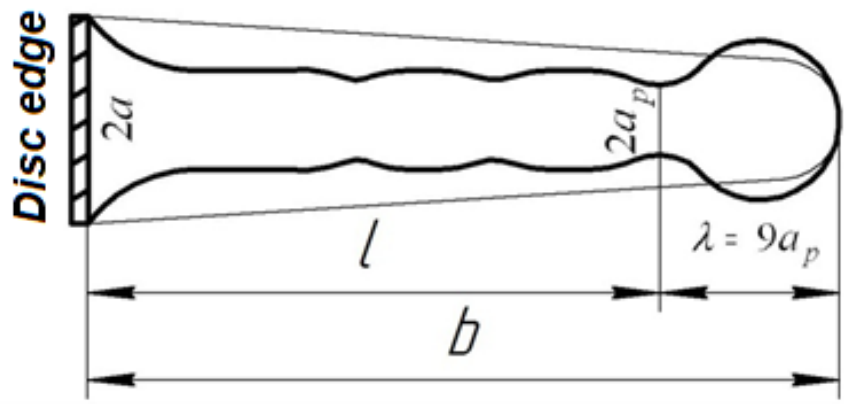

Figure 2. Scheme of fluid thread

The electric field of the generator, in the absence of the bulk charge, is completely defined by the chosen system of electrodes and the form of voltage.

The bulk charge, emerging in the ambient space during electroaerosol generation, distorts the electric field in the area of drops charging, thus exerting a locking action on convectional current, lowering the efficiency of drops charging.

The system of initial equations, describing the electric field of the electro-aerosol generator, can be represented in the form of:

$$
\begin{gathered}
\operatorname{div} E=\frac{\rho}{\varepsilon_{0} \varepsilon} ; \\
\operatorname{div} j_{k}=\operatorname{div}(\rho v)=\frac{\partial \rho}{\partial t} ; \\
m \frac{d v}{d t}=q E+\frac{k_{c}}{b_{r}}(v-w),
\end{gathered}
$$

where: $E$ - intensity of the electric field of the generator, $\mathrm{V} / \mathrm{m}$; $\rho$-bulk charge, $\mathrm{C} / \mathrm{m}^{3} ; v$ - particle rate, $\mathrm{m} / \mathrm{s} ; q$-particle charge, $\mathrm{C} ; b_{r}$ - particle mobility, $\mathrm{m}^{2} /(\mathrm{V} \cdot \mathrm{s}) ; m$ - particle mass, $\mathrm{kg} ; w$-airflow rate, $\mathrm{m} / \mathrm{s} ; k_{c}$ - approximation coefficient of medium resistance.

Let us define the distribution of the bulk charge. For stationary process $\partial \rho / \partial t=0$; then equation of conservation of electric charge (4), for cylindrical electrodes is rearranged in the form of:

$$
\frac{1}{R} \frac{\partial \rho v}{\partial R}=0
$$

or,

$$
\frac{1}{R}\left(v \frac{\partial \rho}{\partial R}+\rho \frac{\partial v}{\partial R}\right)=0
$$


where: $v=\left(w+v_{0}\right) \cdot R_{0} / R ; R_{0}-$ the radius of the inner electrode, m.

Solving equation (7), one obtains the following:

$$
\frac{1}{R}\left[\left(w+v_{0}\right) \frac{R_{0}}{R} \frac{\partial \rho}{\partial R}+\rho\left(w+v_{0}\right) R_{0}\left(-\frac{1}{R^{2}}\right)\right]=0
$$

or,

$$
\frac{\partial \rho}{\partial R}=\frac{\rho}{R}
$$

Given $\rho=\rho_{0}$ and $R=R_{0}$, one obtains:

$$
\rho=\rho_{0} \frac{R_{0}}{R}
$$

where:

$$
\rho_{0}={\frac{I_{\kappa}}{\left(w+v_{0}\right) F_{0}}}^{- \text {initial density of the bulk charge, }}
$$

$\mathrm{C} / \mathrm{m}^{3}$;

$F_{0}$ - the area of the inner electrode, $\mathrm{m}^{2} ; I_{k}$ - convection current, A.

Taking into account (10), equation (3) will take the following form:

$$
\frac{1}{R} \frac{\partial E R}{\partial R}=\frac{\rho_{0} R_{0}}{\varepsilon_{0} \varepsilon R}
$$

Let us rearrange expression (11) in the form of:

$$
\frac{1}{R}\left(R \frac{\partial E}{\partial R}+E \frac{\partial R}{\partial R}\right)=\frac{\rho_{0} R_{0}}{\varepsilon_{0} \varepsilon R} .
$$

Solving linear equation (12) with the use of integral condition $U=\int_{R_{0}}^{R} E d R$ and assuming that $R_{0}=R$, one obtains:

$$
E=\frac{U \varepsilon_{0} \varepsilon_{1}-\rho_{0}\left[R R_{1} \ln R_{1} / R-R_{1}\left(R_{1}-R\right)\right]}{\varepsilon_{0} \varepsilon_{1} R_{1} \ln R_{1} / R} .
$$

For a particular case, $\rho=\rho o$ :

$$
E=\frac{2 U \varepsilon_{0} \varepsilon_{1}-\rho\left[\left(R_{1}^{2}-R^{2}\right) / 2-R^{2} \ln R_{1} / R\right]}{2 \varepsilon_{0} \varepsilon_{1} R \ln R_{1} / R} .
$$

where: $R$ and $R_{l}$ - correspondingly the radii of the generator and a positive electrode, $\mathrm{m}$.
From expressions (13) and (14), it is evident that the intensity of the generator electric field depends on the configuration of the electrodes, applied voltage and bulk charge, emerging during electro-aerosol generation.

Expression (14) can be rearranged in the form of:

$$
E=\frac{2 U \varepsilon_{0} \varepsilon_{1}-\rho \alpha^{\prime}}{2 \varepsilon_{0} \varepsilon_{1} \beta^{\prime}}
$$

where:

$$
\alpha^{\prime}=\frac{R_{1}^{2}-R^{2}}{2}-R^{2} \ln \frac{R_{1}}{R} ; \quad \beta^{\prime}=R \ln \frac{R_{1}}{R} .
$$

From the theory of streams disintegration (Weber, 1931; Levich, 1958), it is known that the charged thread disintegrates in places of deformations, falling behind each other at a distance equal to "wave length" $\lambda$ (figure 2).

For low-viscous fluid:

$$
\lambda=9 a_{p}
$$

where: $\quad a_{p}-$ the stream radius in the place of disintegration.

The time of stream disintegration into drops (theoretically the time of an $e$-time increase of the amplitude of axisymmetric disturbances) is:

$$
t=8,5 \sqrt{\frac{\rho_{\varkappa} a_{p}^{3}}{\alpha}} .
$$

The stream radius in the place of disintegration for the perforated disc equals (Dunsky et al, 1973):

$$
a_{p}=0,39\left(\frac{Q_{\varkappa} \alpha}{\rho_{\varkappa} \omega^{3} n r_{1} R^{3 / 2}}\right)^{2 / 7} .
$$

The length of the nondisintegrated section of the thread amounts to:

$$
l=0,5 R \omega^{2} t^{2}
$$

The number of threads, flowing out from the edge of the generator's holes, is:

$$
K=\frac{2 \pi r_{1} n}{\lambda}
$$

The electric field of the generator exerts electrostatic pressure on fluid streams. 
Capillary pressure of the charged drop on the generator electrode is:

$$
P_{\alpha \ni}=P_{\alpha}-P_{\ni}
$$

The average value of electrostatic pressure on threads, using the expressions of the electric field intensity on the thread surface (Naizade, 1966), can be obtained in the form of:

$$
P_{\ni}=\varepsilon_{0} \beta^{2} E^{2}\left(\eta_{0}^{2}-1\right)
$$

Capillary pressure on the surface of the uncharged thread for the case of $b \gg a$ can be written in the form of:

$$
P_{\alpha}=\alpha \frac{1}{r_{1}}=\frac{\alpha}{a}
$$

Substituting (22) and (23) in (21), the following will be obtained:

$$
\alpha_{\text {э }}=\alpha-\varepsilon_{0} a \beta^{2} E^{2}\left(\eta_{0}^{2}-1\right)
$$

The average radius of the drop during mechanical atomization by the perforated cage (13) is:

$$
r=0,74\left(\frac{Q_{\varkappa}}{R^{3 / 2} \rho_{\varkappa} \omega^{3} n r_{1}}\right)^{2 / 7} \alpha^{2 / 7}
$$

In the electric field with the use of (24), it is:

$$
r_{\ni}=r-\left(\frac{\varepsilon_{0} a \beta^{2} E^{2} r^{7 / 2}\left(\eta_{0}^{2}-1\right)}{\alpha}\right)^{2 / 7},
$$

where: $\quad R-$ the cage radius;

$n, r_{I}-$ correspondingly, the number and the radius of cage's holes.

The charge of the elongated hemisphere (figure 1) in the uniform field equals (Naizade, 1966):

$$
q=q_{n p}(1-\exp (-t / \tau))
$$

where: $q_{n p}=\pi a_{p}^{2} \varepsilon_{0} E \beta$, for the case of $\gamma_{2}>>\gamma_{1}$ and $\varepsilon_{1}=1$

$$
\begin{gathered}
\beta=\frac{1}{\left(\eta_{0} \operatorname{arctn} \eta_{0}-1\right)\left(\eta_{0}^{2}-1\right)} ; \\
\tau=\left[\frac{\varepsilon_{2}}{\gamma_{2}}+\frac{1}{\gamma_{2}\left(\eta_{0} \operatorname{arctn} \eta_{0}-1\right)\left(\eta_{0}^{2}-1\right)}\right] \varepsilon_{0} ;
\end{gathered}
$$

$\eta_{0}=b / c ; \quad c=\sqrt{b^{2}-a_{p}^{2}} ; t-$ the time of streams disintegration into drops, $\mathrm{s} ; b$ - the length of the hemisphere, $\mathrm{m}$; $\varepsilon_{0}-$ the electric constant, $\mathrm{F} / \mathrm{m} ; E-$ the electric field intensity, $\mathrm{V} / \mathrm{m} ; \gamma_{1}$ and $\gamma_{2}$ - correspondingly specific bulk conductance of the air and fluid, $(\mathrm{Ohm} \cdot \mathrm{m})^{-1} ; \varepsilon_{1} \varepsilon_{2}-$ relative dielectric permittivity of the air and fluid.

Surface charge density, taking into account (27):

$$
\sigma_{s}=\frac{q}{s_{u}}=\frac{q}{9 a_{p} \cdot 2 \pi a_{p}+2 \pi a_{p}^{2}}=\frac{q_{n p}(1-\exp (-t / \tau))}{20 \pi a_{p}^{2}}
$$

Let us determine the rate of formation of a new cylindrical surface:

$$
\frac{d S}{d t}=\frac{d 2 \pi a_{p} l}{d t}=2 \pi a_{p} \frac{d l}{d t}
$$

Taking into consideration (19), the following takes place:

$$
\frac{d S}{d t}=\pi a_{p} R \omega^{2} 2 t
$$

Substituting (17) and (18) in (30), the following will be obtained:

$$
\frac{d S}{d t}=1,61 \pi R \omega^{2}\left(\frac{\rho_{\varkappa}}{\alpha}\right)^{1 / 2}\left(\frac{Q_{\varkappa} \alpha}{\rho_{\varkappa} \omega^{3} n r_{1} R^{3 / 2}}\right)^{5 / 7} .
$$

Substituting (31) and (28) in (2), taking into account (15) and supposing that $\rho=\frac{q Q_{\varkappa}}{Q_{B}}$ and $\varepsilon_{1}=1$, the following will obtained:

$$
I_{k}=\frac{2 \varepsilon_{0} U}{\frac{40 \beta^{\prime}}{K \beta m(1-\exp (-t / \tau)}+\frac{\alpha^{\prime}}{Q_{b}}},
$$

where: $\quad m=1,61 \pi R \omega^{2}\left(\frac{\rho_{\varkappa}}{\alpha}\right)^{1 / 2}\left(\frac{Q_{\varkappa} \alpha}{\rho_{\mathscr{K}} \omega^{3} n r_{1} R^{3 / 2}}\right)^{5 / 7}$.

Thus, the simplified mathematical model of electro-aerosol generation and its basic function - convection current - was proposed.

To verify theoretical calculations, experimental studies of convection current of the electro-aerosol generator were undertaken. 
The collector, used in the measuring apparatus, can introduce a number of errors in the results of convection current measurements, which are conditioned by the following factors:

1) the screening of the generator electric field and the bulk charge by the electric field of the collector;

2) auxiliary leakage currents, caused by precipitation of charged particles on the positive electrode of the generator in the collector's field;

3) conduction currents through the electro-aerosol to the collector.

All enumerated factors depend on the distance between the positive electrode of the generator and the collector, as well as on their configuration and sizes, that is, on capacity $C_{2}$ (figure 3 ).

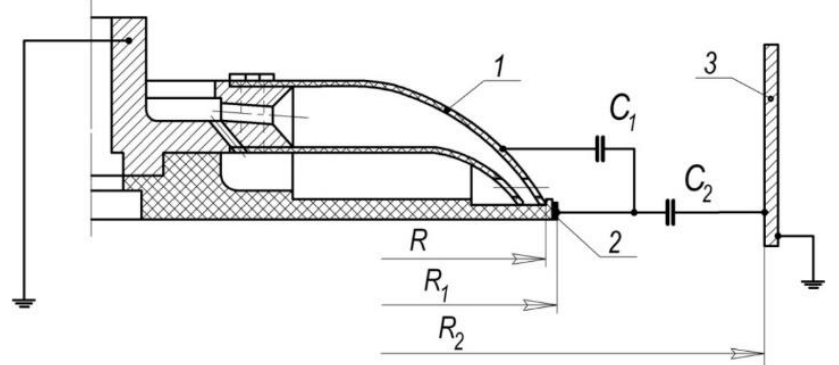

Figure 3. Scheme of electrodes of generator and collector

1 - grounded generator electrode; 2 - positive electrode of the generator;

3 - collector; $C_{l}$ - capacity among generator's electrodes; $C_{2}$ - capacity between the positive electrode of the generator and the collector.

From obvious considerations, it is clear that an increase of capacity $C_{2}$ higher than capacity $C_{l}$ will cause the screening of the generator's field and a decrease of current $I_{c}$. When $C_{1} \geq C_{2}$, the influence of the bulk charge in the generator's field decreases and $I_{c}$ increases. A similar analysis can be conducted using points 2 and 3. Thus, to prevent the collector's influence on convection current, the following condition must be fulfilled:

$$
C_{1} \gg C_{2}
$$

For the scheme, shown in figure 3, with a cylindrical system of electrodes, let us write (33) in the form of:

$$
\frac{2 \pi \varepsilon_{0} l}{\ln \left(R_{1} / R\right)}>>\frac{2 \pi \varepsilon_{0} l}{\ln \left(R_{2} / R_{1}\right)} ;
$$

or,

$$
\ln \frac{R_{2}}{R_{1}}>>\ln \frac{R_{1}}{R}
$$

where: $\quad l$ - length of the positive electrode, $\mathrm{m}$;

$R, R_{1}, R_{2}$ - correspondingly radii of the atomizing element, the positive electrode, the collector, $\mathrm{m}$.

Substituting the sizes of the generator and the collector in (35), the following will be obtained:

$$
\ln \frac{0,5}{0,163}>>\ln \frac{0,163}{0,16}
$$

or,

$$
1,12 \gg 0,0185 \text {. }
$$

Condition (33) is fulfilled; therefore, the collector's diameter, equal to $1 \mathrm{~m}$, used in measurements, can be considered to be chosen correctly.

The results of the experimental studies and dependences, calculated according to expression (32), are presented in figure 4 .

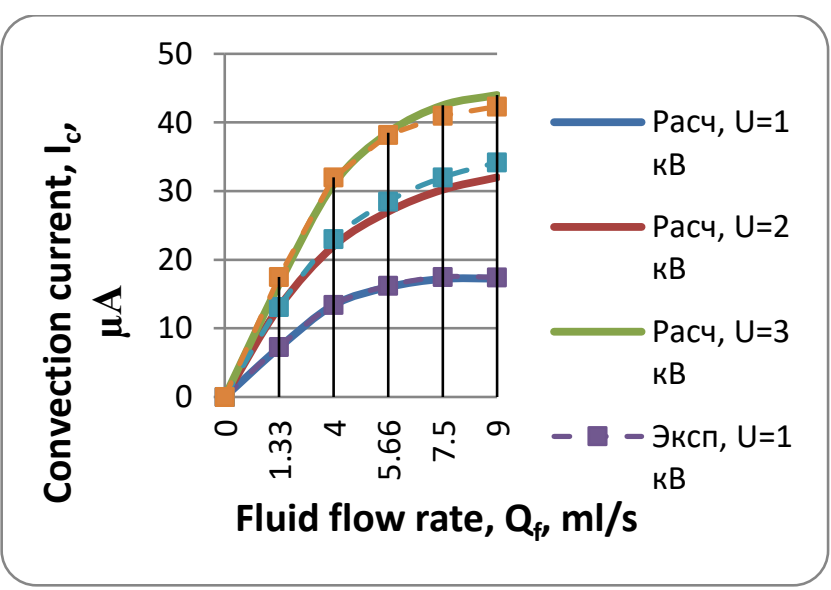

Figure 4. Dependence of convection current $I_{c}$ on fluid flow rate $Q_{f}$ at different voltage $U$

$\begin{array}{lll}\text { Calc, } \mathrm{U}=1 \mathrm{kV} & ; & \text { Calc, } \mathrm{U}=2 \mathrm{kV} \\ \text { Calc, } \mathrm{U}=3 \mathrm{kV} & ; & \text { Exp, } \mathrm{U}=1 \mathrm{kV} \\ \text { Exp, } \mathrm{U}=2 \mathrm{kV} & ; & \text { Exp, } \mathrm{U}=3 \mathrm{kV}\end{array}$

\section{CONCLUSIONS}

A simplified mathematic model of electro-aerosol generation and its basic function - convection current - was proposed.

Figure 2 shows that convection current increases when increasing the fluid flow rate and the charging voltage. Nonlinearity of dependences is explained by the influence of specific charge on the process of aerosol charging.

From the obtained dependences, it is obvious that experimental and theoretical curves have good convergence, which allows 
using expression (32) for calculation of the parameters of electroaerosol generators during their designing.

\section{RECOMMENDATIONS}

The proposed mathematical model of electro-aerosol generation was obtained for the generator of mechanical type with perforated holes on the grounded electrode with the use of inductive charging. The generator's mode of operation, in which the fluid torus, from which separate threads are drawn out, forms at the edge of the disc, is accepted.

For other types of atomizing discs, one should take into account the perimeter of the disc edge, from which atomization of the fluid film takes place. With low fluid flow rates, the fluid torus, formed at the disc edge, can disintegrate into separate drops without forming a fluid thread (Pazhi and Galustov, 1984; Dunsky and Nikitin, 1982). In this case, it is necessary to consider the drop charge in the electric field, but not an elongated hemisphere.

\section{REFERENCES}

Bals, E. J., 1970. Rotary atomization. Agr. Aviat, 12(3), pp. 8590.

Blyumin G.Z., Astapov S.V., 1987. Compressorless aerosolization of premises. Pigbreeding (Svinovodstvo), 3, p 32.

Borodin I.F., Bukharin I.L., Lekomtsev P.L., 2004. Abating the sources of microbial contamination. Rural Mechanic (Selskiy mekhanizator), 1, pp. 20.

Borodin I.F., Lekomtsev P.L., 2006. Study of electro-aerosol dissemination in large-scale livestock premises. Reports of RAAS (Doklady RASHN), 4, pp. 53-55.

Borodin I.F., Lekomtsev P.L., 2005. New electro-aerosol generators for agricultural production. Herald of Moscow SAU n. a. V.P. Goryachkin (Vestnik Moskovskogo GAU im. V.P. Goryachkina), 3 (13), pp. 15.

Borodin V.A., Ditaytkin Yu.F., Klyachko L.A., 1967. Atomization of fluids. Mechanical Engineering (Mashinostroyeniye), Moscow.

Borok A.M., 1974. Analysis of operation of electrostatic atomizer and sources of charges of EHD-generators with consideration of particle mobility. Tr. of ChIMEA ( $\mathrm{Tr}$. CHIMESH), issue 85. CHIMESH, Chelyabinsk.

Dondokov D.D., Tumureev N.V., 1976. Dynamics of precipitation of uniporlarly charged aerosol indoors, taking into account its hermeticity. Tr. ChIMEA (Tr. CHIMESH), Iss. 110, CHIMESH, Chelyabinsk.
Dunsky V.F., 1966. Inductive method of unipolar electrization during formation of aerodisperse systems. Colloid Journal (Kolloidny zhurnal), vol. XXVIII, 1, pp. 34-38.

Dunsky V.F., Kitaev A.V., 1960. Precipitation of uniporlarly charged aerosol indoors. Colloid Journal (Kolloidny zhurnal), vol. XXII, 2.

Dunsky V.F., Nikitin N.V., 1982. Mechanical atomization of fluids, in: "Aerosols' Role in Plant Protection". VASHNIL, Ear (Kolos), Moscow.

Dunsky V.F., Nikitin N.V., Sokolov M.S., 1973. Monodisperse Aerosols. Science (Nauka), Moscow.

Frost A. R., 1974. Rotary atomization. Brit. Crop. Prot. Coun. Monogr, vol. 11, pp. 120-127.

Green H., Lane W., 1972. Aerosols - Dusts, Smokes, and Mists. 2nd Ed., Chemistry (Khimiya), Moscow.

Hinze, Y. O, Milborn H., 1950. Atomization of liquids by means of a rotating cup, J. Appl. Mech, vol. 17(2), pp. 145-153.

Law, S. E., 1975. Theoretically predicted interaction of surface charge and evaporation in airborne pesticide droplets. Trans. of the ASAE.

Lekomtsev P.L., 2006. Electro-aerosol technologies in agriculture: monograph. FSEI HPE Izhevsk GAA (FGOU VPO Izhevskaya GSHA), Izhevsk.

Levich V.G., 1958. Physical-Chemical Hydrodynamics. Fizmatgiz, Moscow.

Makalsky L.M., 1991. Generation and use of charged electroaerosols. Application of electronic-ionic technology in national economy: Theses of Reports of IV All-Union Conference. Moscow, pp. 202-215.

Naizade A.T., 1966. Charging of particles of elongated shape on flat electrode. Proc. of AS USSR. Power Engineering and Transport (Energetika i transport), 1.

Pazhi D.G., Galustov V.S., 1984. Basics of Technology of Fluid Atomization. Chemistry (Khimiya), Moscow.

Savushkin A.V., Lekomtsev P.L., 1991. Experimental research of convection current and aerosol during mechanical-air atomization. Application of Electric Energy in Agriculture: Collected Papers of MIIAP, pp. 19-21.

Savushkin A.V., Lekomtsev P.L., Trofimov N.V., 1991. Electroaerosol generator tester. Application of Electric Energy in Agriculture: Collected Papers of MIIAP. MIIAP, Moscow. 
Vereshchagin I.P., Levitov V.I., Mirzabekyan G.Z., Pashin M.M., 1974. Basics of Electrogasdynamics of Disperse Systems. Energy (Energiya), Moscow.

Weber, K. Z., 1931. Angem. Math. und Mech, 11, pp.13.

Yarnyh, V.S., 1972. Aerosols in Veterinary. Ear (Kolos), Moscow.

Yarnyh, V.S., 1985. Condition and prospects of veterinarysanitary science development. Tr. of ASRIEER (Tr. VNIIVS), Moscow, pp. 3-15.

Zakomyrdin A.A., Visnapuu L.Yu., 1977. Decontamination of livestock premises by aerosols of disinfection substances. In: Theses of the 3rd All-Union Conf. on Aerosols. vol. 3. Moscow, pp. $41-42$. 